
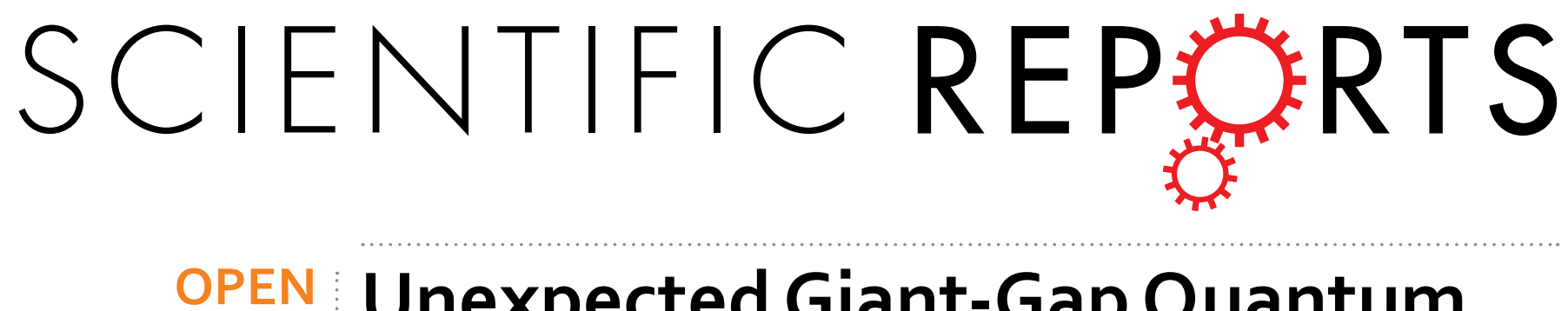

\title{
Unexpected Giant-Gap Quantum Spin Hall Insulator in Chemically Decorated Plumbene Monolayer
}

Received: 05 August 2015

Accepted: 30 December 2015

Published: 02 February 2016
Hui Zhao ${ }^{1}$, Chang-wen Zhang ${ }^{1}$, Wei-xiao $\mathrm{Ji}^{1}$, Run-wu Zhang ${ }^{1}$, Sheng-shi Li ${ }^{2}$, Shi-shen Yan ${ }^{2}$, Bao-min Zhang ${ }^{1}$, Ping Li ${ }^{1}$ \& Pei-ji Wang ${ }^{1}$

Quantum spin Hall (QSH) effect of two-dimensional (2D) materials features edge states that are topologically protected from backscattering by time-reversal symmetry. However, the major obstacles to the application for QSH effect are the lack of suitable QSH insulators with a large bulk gap. Here, we predict a novel class of 2D OSH insulators in X-decorated plumbene monolayers ( $\mathrm{PbX} ; \mathrm{X}=\mathrm{H}, \mathrm{F}, \mathrm{Cl}$, $\mathrm{Br}, \mathrm{I})$ with extraordinarily giant bulk gaps from $1.03 \mathrm{eV}$ to a record value of $1.34 \mathrm{eV}$. The topological characteristic of $\mathrm{PbX}$ mainly originates from $s-p_{x, y}$ band inversion related to the lattice symmetry, while the effect of spin-orbital coupling (SOC) is only to open up a giant gap. Their QSH states are identified by nontrivial topological invariant $Z_{2}=1$, as well as a single pair of topologically protected helical edge states locating inside the bulk gap. Noticeably, the QSH gaps of $\mathrm{PbX}$ are tunable and robust via external strain. We also propose high-dielectric-constant $\mathrm{BN}$ as an ideal substrate for the experimental realization of $\mathrm{PbX}$, maintaining its nontrivial topology. These novel $\mathrm{QSH}$ insulators with giant gaps are a promising platform to enrich topological phenomena and expand potential applications at high temperature.

Topological insulators (TIs) ${ }^{1-4}$, as a new class of quantum materials, have generated intensive research activities in quantum information, because they give an alternative and robust platform for obtaining relativistic and spin-polarized Fermions in the condensed matter system. One of the most interesting phenomena in this realm is the quantum spin Hall (QSH) effect in two-dimensional (2D) materials ${ }^{1,2}$, characterized by an insulating bulk-gap and gapless edge states at its boundaries due to time-reversal symmetry (TRS), thus providing the enticing concepts for novel quantum electronic devices with low energy dissipation ${ }^{3,4}$. The prototypical concept of QSH insulator is first proposed by Kane and Mele in graphene $e^{5,6}$, in which the spin-orbit coupling (SOC) opens a band gap at the Dirac point. However, the associated gap due to rather weak second-order effective SOC is too small $\left(\sim 10^{-3} \mathrm{meV}\right)$, which makes the QSH state in graphene only appear at an unrealistically low temperature. Quantized conductance through QSH edge states have only been experimentally demonstrated in $\mathrm{HgTe} / \mathrm{CdTe} \mathrm{C}^{7,8}$ and $\mathrm{InAs} / \mathrm{GaSb}^{9,10}$ quantum wells at the ultralow temperature, which greatly obstructs their potential applications in spintronics.

To be "good" QSH insulators, the materials should have a large bulk-gap to realize the spin-polarized electron transport at room temperature. Controlling the chemical bonding at atomic levels to induce the band inversion ${ }^{3,4}$ by SOC is an efficient way to realize topologically nontrivial $\mathrm{Z}_{2}$ number ${ }^{8,11-15}$. The $2 \mathrm{D}$ thin films are advantageous in this aspect as their bonding properties are easy to be modified in post synthesis processes, for example, by surface adsorption to enlarge SOC strength. Group-IV honeycomb lattices such as hydrogenated or halogenated germanene and stanene ${ }^{16,17}$ monolayers have been reported as a QSH phase with a bulk-gap as large as $0.2-0.3 \mathrm{eV}$. However, most QSH phases in these systems are not intrinsic, but driven by external strain, thus are unfavorable for practical applications in spintronics. On the other hand, Rivero et al. ${ }^{18}$ recently reported the stability and properties of high-buckled 2D Sn and $\mathrm{Pb}$ thin films. They find that the optimal structure of fluorinated stanene lacks threefold symmetry and thus the experimental realization of $2 \mathrm{D}$ fluorinated one is challengeable. The strong SOC effect can also be sufficed in group-V heavy elements such as bismuth, which drives a nontrivial QSH state ${ }^{19}$. Besides, an approach to design a large-gap QSH state on a semiconductor surface by a substrate orbital filtering

${ }^{1}$ School of Physics and Technology, University of Jinan, Jinan, Shandong, 250022, People's Republic of China. ${ }^{2}$ School of Physics, State Key laboratory of Crystal Materials, Shandong University, Jinan, Shandong, 250100, People's Republic of China. Correspondence and requests for materials should be addressed to C.-W.Z. (email: zhchwsd@163. com) 
(a)

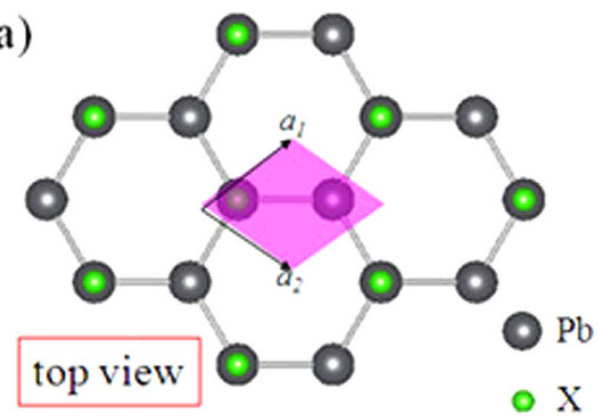

(b)

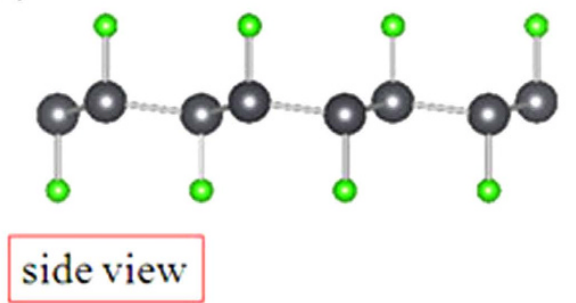

(c)

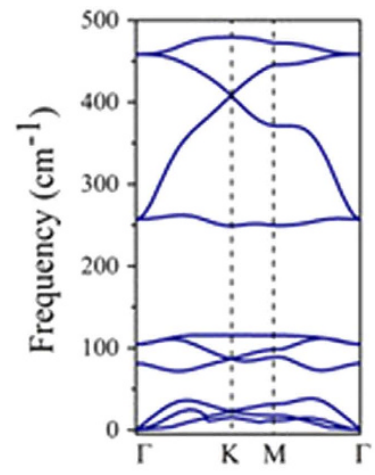

(d)

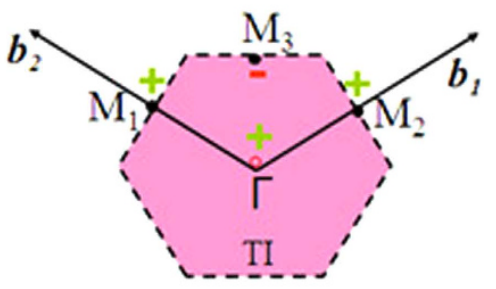

Figure 1. (a,b) Top and side views of the schematic structures of $\mathrm{PbH}$ monolayer. Black and green balls denote $\mathrm{Pb}$ and $\mathrm{H}$ atoms, respectively. Shadow area in (a) present the unit cell. (c) Phonon band dispersions, and (d) the area of Brilioun zone of $\mathrm{PbH}$ monolayer.

\begin{tabular}{|l|c|c|c|c|c|c|c|c|}
\hline monolayers & $\mathbf{a}(\AA)$ & $\mathbf{d}_{\mathbf{1}}(\AA)$ & $\mathbf{d}_{\mathbf{2}}(\AA)$ & $\boldsymbol{\Delta}(\AA)$ & $\mathbf{v}_{\mathbf{f}}\left(\mathbf{1 0} \mathbf{5}^{\mathbf{m}} / \mathbf{s}\right)$ & $\boldsymbol{E}_{\mathrm{g}}{ }^{\mathbf{P B E}}(\mathbf{e V})$ & $\boldsymbol{\Delta} \boldsymbol{E}_{f}(\mathbf{e V} / \mathbf{a t o m})$ & $\mathbf{Z}_{\mathbf{2}}$ \\
\hline $\mathrm{Pb}$ & 4.9257 & 2.9906 & $\backslash$ & 0.9256 & $\backslash$ & 0.421 & $\backslash$ & 0 \\
\hline $\mathrm{PbH}$ & 5.0359 & 3.0098 & 1.8365 & 0.7781 & 2.5616 & 0.983 & -1.89 & 1 \\
\hline $\mathrm{PbF}$ & 5.1431 & 2.9902 & 2.0888 & 0.3518 & 1.2472 & 1.071 & -2.82 & 1 \\
\hline $\mathrm{PbCl}$ & 5.3017 & 3.1463 & 2.4934 & 0.4161 & 0.7215 & 0.902 & -1.48 & 1 \\
\hline $\mathrm{PbBr}$ & 5.3795 & 3.1424 & 2.6362 & 0.4779 & 0.6573 & 0.847 & -1.32 & 1 \\
\hline $\mathrm{PbI}$ & 5.4441 & 3.1346 & 2.8394 & 0.5528 & 0.4961 & 0.693 & -0.86 & 1 \\
\hline
\end{tabular}

Table 1. The present crystal parameters include the lattice constant $\mathrm{a}, \mathrm{Pb}-\mathrm{Pb}$ bond length $\mathrm{d}_{1}, \mathrm{~Pb}-\mathrm{X}$ bond length $\mathrm{d}_{2}$, buckling height $\Delta$ ( $\Delta$ is defined as the distance from the center of the upper to that of the lower $\mathrm{Pb}$ atoms), and the global band gap $E_{\mathrm{g}}{ }^{\mathrm{PBE}}$ calculated with $\mathrm{PBE}$, as well as the formation energy $\Delta \mathrm{E}_{\mathrm{f}}$. The Fermi velocity is shown as $\mathrm{v}_{\mathrm{f}}$, and topological invariant is defined as $\mathrm{Z}_{2}$.

process is also proposed ${ }^{20,21}$. More recently, the ethynyl-functionalized stanene has been reported to be a good QSH insulator ${ }^{22}$. These large-gap QSH insulators are essential for realizing many exotic phenomena and for fabricating new quantum devices that can operate at room temperature.

In this work, on the basis of first-principles calculations, we predicted a class of new QSH insulators in $\mathrm{X}$-decorated plumbene monolayers $\left(\mathrm{PbX} ; \mathrm{X}=\mathrm{H}, \mathrm{F}, \mathrm{Cl}, \mathrm{Br}\right.$, and $\mathrm{I}$ ), in analogy 2D hydrocarbon (graphane) ${ }^{23,24}$. An extraordinarily nontrivial giant-gap in the range of $1.03 \sim 1.34 \mathrm{eV}$ were obtained, making the experimental observation of QSH phase facile. The topological characteristic of $\mathrm{PbX}$ was attributed to $s-p_{x, y}$ band inversion related to the lattice symmetry, while the effect of SOC was only to open up a giant-gap. Interestingly, the QSH gaps of $\mathrm{PbX}$ were tunable and robust against external strain. Our findings had potential applications in low-power quantum electronics and may enable topological quantum computing based on Majorana fermions ${ }^{25}$.

Figure 1 (a) shows the typical lattice structure of $2 \mathrm{D} \mathrm{PbX}$, in which the $\mathrm{Pb}$ atoms are in $\mathrm{sp}^{3}$ hybridization forming a hexagonal network, along with $\mathrm{X}$ being bonded to $\mathrm{Pb}$ atoms on both sides of the plane in an alternating manner. In comparison with the pristine plumbene in Fig. $\mathrm{S} 1$, all $\mathrm{Pb}-\mathrm{Pb}$ bonds in $\mathrm{PbX}$ slightly expand, while the buckling decreases clearly due to the weakly hybridization between $\pi$ and $\sigma$ orbitals, as listed in Table 1 . We also note the similar results in halogenated germanene and stanene monolayers ${ }^{16,17}$. The buckling plays a crucial role in the engineering of the band structures in these materials. The high structural stability is verified by the formation energy defined as

$$
\Delta E_{f}=E(P b X)-E(P b)-1 / 2 E\left(X_{2}\right)
$$

where $E(\mathrm{PbX})$ and $E(\mathrm{~Pb})$ are the total energies of decorated and pristine plumbene, respectively, while $E\left(\mathrm{X}_{2}\right)$ is the chemical potential of hydrogen or halogen molecules. The calculated formation energy $\Delta E$ is found to be -1.89 , $-2.82,-1.48,-1.32$, and $-0.86 \mathrm{eV} /$ atom for $\mathrm{PbH}, \mathrm{PbF}, \mathrm{PbCl}, \mathrm{PbBr}$, and $\mathrm{PbI}$, respectively, indicating no phase 

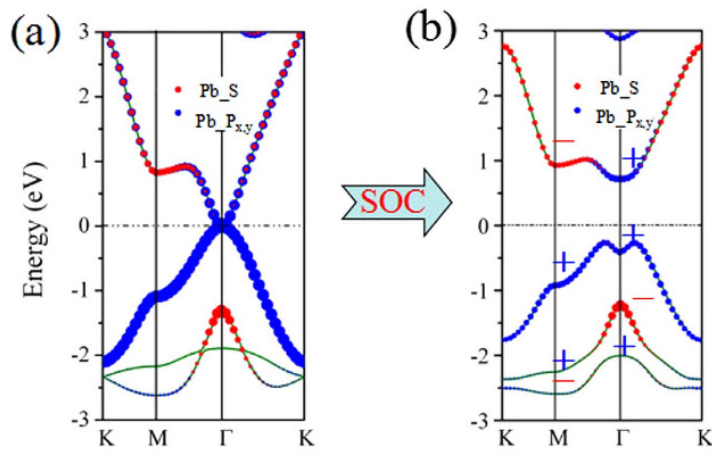

(c)

\begin{tabular}{|c|c|c|}
\hline$\Gamma_{\mathrm{i}}$ & Parity of $\zeta_{2 \mathrm{n}}$ of occupied bands & \\
\hline$(0,0)$ & $+\ldots+\ldots+$ & + \\
\hline$(0.5,0)$ & $+\ldots++$ & + \\
\hline$(0,0.5)$ & $+\ldots++$ & + \\
\hline$(0.5,0.5)$ & -++- & - \\
\hline $\mathrm{PbH}$ & $\mathrm{Z}_{2}$ topological invariant & $\mathrm{v}=1$ \\
\hline
\end{tabular}

Figure 2. Band structures for $\mathbf{P b H}$ without SOC (a) and with SOC (b) with zooming in the energy dispersion near the Fermi level. The red circles and blue squares represent the weights of the $\mathrm{Pb}-s$ and $\mathrm{Pb}-\mathrm{p}_{x, y}$ character, respectively. (c) Parities of occupied spin-degenerate bands at the TRIM Points for $\mathrm{PbH}$. Here, we show the parities of 5 occupied spin-degenerate bands for $\mathrm{PbH}$. Positive and negative signs denote even and odd parities, respectively.

separation in these systems. Besides, we also calculate the phonon dispersion for $\mathrm{PbX}$ monolayers, as illustrated in Fig. 1(c) and Fig. S2. The frequencies of all modes are positive over the whole Brillouin zone, implying that $\mathrm{PbX}$ are thermodynamically stable.

We now focus on the band structures of $\mathrm{PbH}$ monolayer as an example. In the absence of SOC (Fig. 2(a)), the band gap at the $\mathrm{K}$ point is substantially enlarged due to the saturation of the $\mathrm{Pb}-p_{z}$, maintaining $p_{x, y}$ level located at the $\Gamma$ point. Thus, $\mathrm{PbH}$ can be regarded as a gapless semiconductor. By further projecting the bands onto different atomic orbitals, we find that the energy spectrums at the $\Gamma$ point mainly come from one $s$ and two $p$ orbitals of $\mathrm{Pb}$ atoms. When the effect of SOC is taken into consideration (Fig. 2(b)), the energy degeneracy of $p_{x, y}$ orbitals at the $\Gamma$ point are lifted, indicating a semimetal-to-semiconductor transition. Away from the $\Gamma$ point, the conduction bands are upshifted, whereas the valence bands are downshifted, which produces a global indirect-gap as large as $0.98 \mathrm{eV}$, ample for applications at room temperature. These suggest that $\mathrm{PbH}$ could be TIs upon opening a band-gap at the touching point by SOC. To further confirm these results, we also have employed HSE06 ${ }^{26}$ to check the electronic structures of $\mathrm{PbH}$ monolayer in Fig. S3, which are in good agreement with these obtained here.

The SOC-induced gap opening near the Fermi level indicates possible existence of 2D TI phase that are helical with the spin-momentum locked by TRS. Thus, we calculate the topological edge states of $\mathrm{PbH}$ by the Wannier90 package ${ }^{27}$. We construct the maximally localized Wannier functions (MLWFs) and fit a tight-binding Hamiltonian with these functions. Figure 3(a) shows the DFT and fitted band structures, in well agreement with each other. Then, the edge Green's function ${ }^{28}$ of a semi-infinite $\mathrm{PbH}$ is constructed and the local density of state (LDOS) of $\mathrm{PbH}$ is calculated, as shown in Fig. 3(c). Clearly, all the edge bands connect completely the conduction and valence bands and span the 2D bulk band gap, yielding 1D gapless edge states. Besides, the counter-propagating edge states exhibit opposite spin polarizations, in accordance with the spin-momentum locking of 1D helical electrons (Fig. 3(d)). On the other hand, we construct a zigzag-edged nanoribbon, as shown in Fig. 3(e). All the edge $\mathrm{Pb}$ atoms are hydrogenated to eliminate the angling bonds. The width of the nanoribbon, $20.6 \mathrm{~nm}$, is large enough to avoid interactions between the edge states of the two sides, and the band structure of the nanoribbon is shown in Fig. 3(f). We can explicitly observe that the gapless edge states (red lines) that appear within the bulk gap and cross linearly at the $\Gamma$ point, demonstrating the topological nontribal property of the bulk gap, which is consistent with the Fig. 3(b). In this case, the Dirac point located at the band gap is calculated to have a high velocity of $\sim 1.0 \times 10^{5} \mathrm{~m} / \mathrm{s}$, larger than that of $3.0 \times 10^{4} \mathrm{~m} / \mathrm{s}$ in $\mathrm{InAs} / \mathrm{GaSb}$ quantum well ${ }^{9,10}$, indicating that $\mathrm{PbH}$ is an ideal $2 \mathrm{D} \mathrm{TI}$.

To further confirm the topological edge states of $\mathrm{PbH}$ monolayer, we calculate the $\mathrm{Z}_{2}$ invariant $v$ based on the method proposed by $\mathrm{Fu}$ and $\mathrm{Kane}^{29}$, due to the presence of structural inversion symmetry. Here, the topological index $v$ can be established by 
(a)

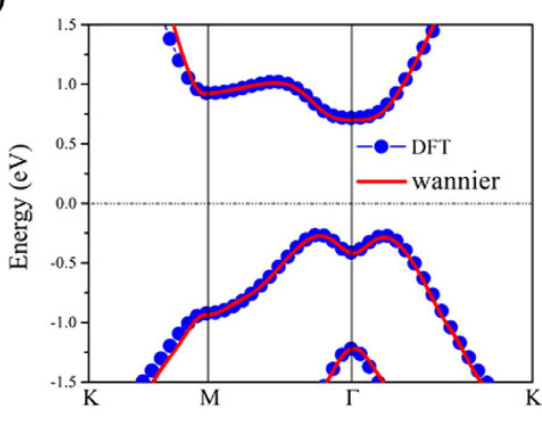

(c)

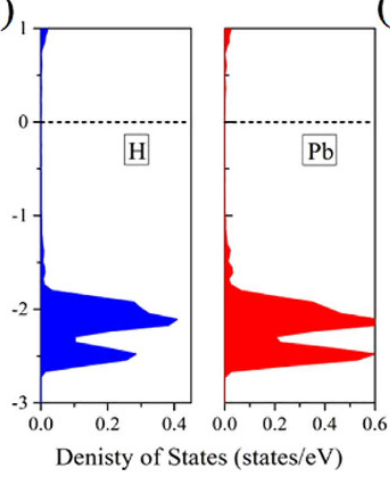

(e)

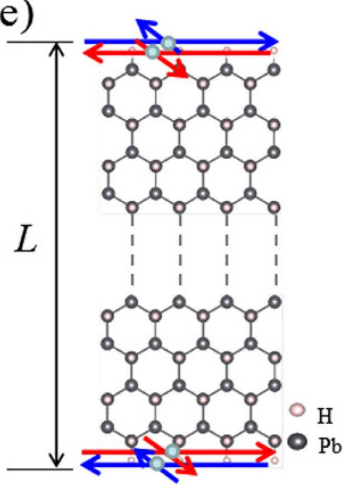

(b)

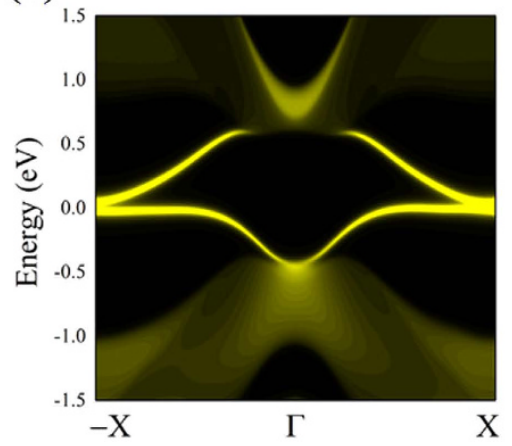

(d)

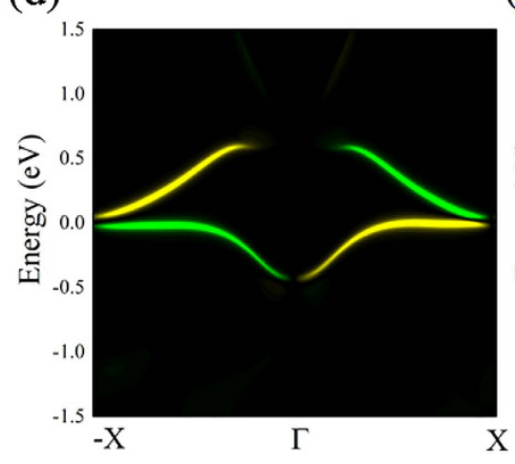

(f) 2

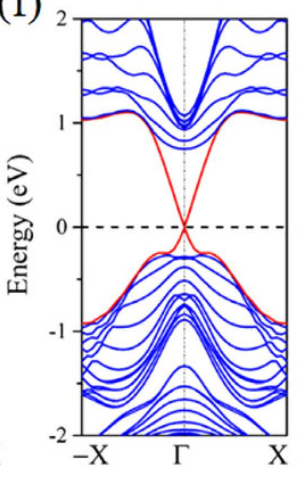

Figure 3. Electronic structures and its corresponding edge state of $\mathrm{PbH}$. (a) Comparison of band structures for $\mathrm{PbH}$ calculated by DFT (red lines) and Wannier function method (blue circles). (c) Illustration of the partial DOS projected onto $p_{z}$ orbital of $\mathrm{Pb}$ and the total $\mathrm{DOS}$ of $\mathrm{H}$ atom. The Fermi level is set to zero. (b,d) show the Dirac edge states, and edge spin polarization, respectively. The Fermi level is set to zero. (e,f) the model and spectrum of a finite slab of $\mathrm{PbH}$. The red and blue horizontal arrows represent the spin-up and -down polarized currents in opposite direction.

$$
\delta\left(K_{i}\right)=\prod_{m=1}^{N} \xi_{2 m}^{i},(-1)^{\nu}=\prod_{i=1}^{4} \delta\left(K_{i}\right)=\delta(\Gamma) \delta(M)^{3}
$$

Where $\delta$ is the product of parity eigenvalues at the time-reversal-invariant momenta (TRIM) points, $\xi= \pm 1$ are the parity eigenvalues and $N$ is the number of the occupied bands. According to the $Z_{2}$ classification, $\nu=1$ characterizes a topologically nontrivial phase and $\nu=0$ means a topologically trivial one. Here, the invariants $Z_{2}$ are derived from the parities of wave function at the four TRIM points $\mathrm{K}_{\mathrm{i}}$, including $\Gamma(0,0), M_{1}(0,1 / 2), M_{2}(1 / 2,0)$, and $M_{3}(1 / 2,1 / 2)$, as illustrated in Fig. 1(d). From the calculated results in Fig. 2(c), we find the $\mathrm{Z}_{2}$ of $\mathrm{PbH}$ monolayer is +1 , indicating that it is a QSH insulator.

To understand the physical origin of QSH state, we next do an orbital analysis around the Fermi level for $\mathrm{PbH}$ monolayer. As can be seen from density of states (DOS) in Fig. 3(c), the decorated $\mathrm{H}$ atoms hybridizes strongly with the dangling bonds of $p_{z}$ orbital in $\mathrm{Pb}$ atoms overlapping in the same energy range, which effectively removes the $p_{z}$ bands away from the Fermi level, leaving only the $s$ and $p_{x, y}$ states at the Fermi level. Thus, we present systematically the band evolution at the $\Gamma$ point for $\mathrm{PbH}$ monolayer in Fig. 4(a). One can see that the chemical bonding and crystal field splitting between $\mathrm{Pb}-\mathrm{Pb}$ atoms make the $s$ and $p_{x, y}$ orbitals split into the bonding and anti-bonding states, i.e., $\left|\mathrm{s}^{ \pm}\right\rangle$and $\left|p_{x, y}{ }^{ \pm}\right\rangle$, which the superscripts + and - represent the parities of corresponding states, respectively. Noticeably, the band orders of $\mathrm{PbH}$ are different from the previous reported cases in $\mathrm{GeH} /$ $\mathrm{SnH}^{16,17}$, where the $\left|p_{x, y}{ }^{+}\right\rangle$lies below $\left|\mathrm{s}^{-}\right\rangle$orbital, which leads to inversion of the bands around the Fermi level, to produce the crucial QSH effect. This is because that the modification of band order upon surface functionalization is related to the bond length and orbital splitting. The larger lattice constant of PbH monolayer $(4.978 \AA)$ results in a weaker $s$ - $p$ hybridization, and accordingly a smaller energy separation between the bonding and antibonding states. Thus, the $\left|\mathrm{s}^{-}\right\rangle$orbital is downshifted while the $\left|p_{x, y}{ }^{+}\right\rangle$orbital is upshifted, i.e., the $\left|\mathrm{s}^{-}\right\rangle$will be occupied, while the quadruply degenerate $\left|p_{x, y}{ }^{+}\right\rangle$is half occupied (due to the $\mathrm{C}_{3}$ rotation symmetry), resulting in semi-metallic character (Fig. 2(a)). With the inclusion of SOC, the degeneracy of the level splits into $|p, \pm 3 / 2\rangle$ state with a total angular momentum $j=3 / 2$ and $|p, \pm 1 / 2\rangle$ with a total angular momentum $j=1 / 2$, opening a full band gap. Obviously, the adsorbed hydrogen atoms act like an orbital filter to selectively remove the $p_{z}$ orbitals from the $\mathrm{Pb}$ lattice, maintaining a nontrivial six-band lattice with $\mathrm{Z}_{2}=1^{30}$. Here, an interesting feature of band structure is that the effect of SOC is only producing an band gap around the Fermi level, but not inducing band 
(a)

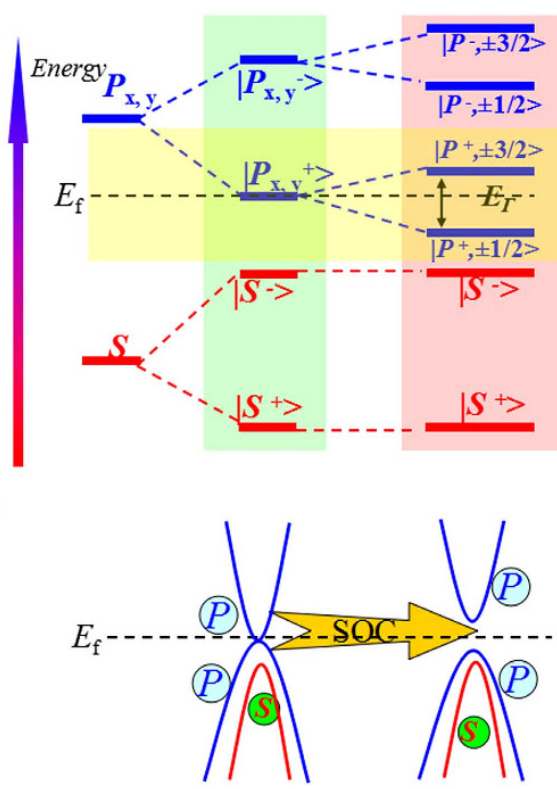

(c)

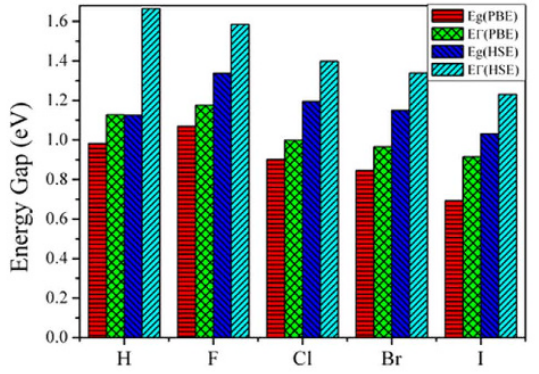

(d)

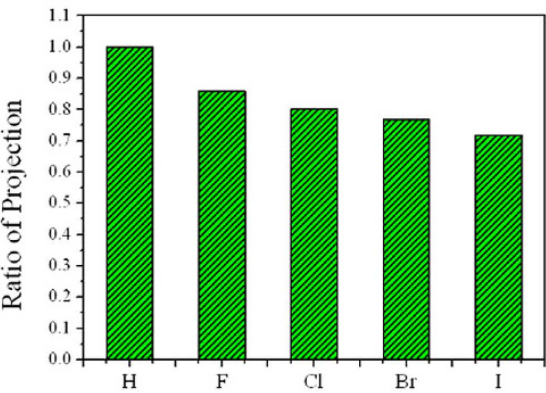

Figure 4. (a) The evolution of atomic $s$ and $p_{\mathrm{x}, \mathrm{y}}$ orbitals of $\mathrm{PbH}$ into band edges at $\Gamma$ point is described as the crystal field splitting and SOC are switched in sequence. (b) Illustration of the effect of SOC on the inversion of bands around the Fermi level. (c) The calculated global band gap by PBE and HSE methods, and (d) the ratio of $\mathrm{Pb}-p_{x, y}$ component in the total orbital at $\Gamma$ point near the Fermi level.

inversion, i.e., SOC is not relevant for the formation of nontrivial band orders. The brand new mechanism here may provide an efficient way to search for large-gap QSH insulators in $2 \mathrm{D}$ materials ${ }^{20,21}$. In fact, a similar situation has even been observed in well-known 2D TIs such as graphene and silicene ${ }^{5,6,12}$, where the inclusion of SOC does not change the band order between $\pi$ and $\pi^{*}$ bands. However, the nontrivial QSH effect of graphene mainly originates from the massive Dirac cone ${ }^{5,6}$, but the QSH phase in $\mathrm{PbH}$ is attributed to $s$ - $p$ band inversion at $\Gamma$ the point, similar to that in the $\mathrm{Bi}_{2} \mathrm{Se}_{3}$ or $\mathrm{HgTe}$ quantum well ${ }^{8,13}$.

Here, it is worth emphasizing that the hydrogenation in plumbene is not the only way to achieve the giant-gap QSH state, the same results can be obtained by decorating the surface with otherwise halogen atoms, such as $\mathrm{F}, \mathrm{Cl}, \mathrm{Br}$, and $\mathrm{I}$. We thus performed calculations for $\mathrm{PbX}(\mathrm{X}=\mathrm{F}, \mathrm{Cl}, \mathrm{Br}, \mathrm{I})$ to check their topological properties, as illustrated in Figs S3-S5. Table 1 summarizes their lattice constants, $\mathrm{Pb}-\mathrm{Pb}$ bond lengths, and nontrivial gaps at their equilibrium states. These results demonstrate that all the electronic structures of halogenated $\mathrm{PbX}$ monolayers are similar to $\mathrm{PbH}$, and exhibit nontrivial topological invariant $\mathrm{Z}_{2}=1$ (Table $\mathrm{S} 1$ ). Noticeably, as can be seen in Fig. 4(c) and Fig. S2, the global QSH gaps of PbF, PbCl, PbBr, and PbI are 1.34, $1.19,1.15$, and $1.03 \mathrm{eV}$, respectively, which are sufficiently large for practical applications at high temperature. On the other hand, we can find some interesting phenomena when comparing the band gaps with each other. It is known that, from $\mathrm{F}$ to $\mathrm{I}$, the SOC becomes stronger in the order of $\mathrm{F}<\mathrm{Cl}<\mathrm{Br}<\mathrm{I}$, thus the SOC induced band gap should follow this trend. Since the band gaps of PbX monolayers are mainly determined by SOC strength near the Fermi level, this order also works. However, the fact is just opposite, where the band gaps of $\mathrm{PbX}$ decrease monotonically, namely, $\mathrm{PbF}>\mathrm{PbCl}>\mathrm{PbBr}>\mathrm{PbI}$. Further explanation of this interesting contradiction can be attributed to the band components of $\mathrm{Pb}$ atoms near the Fermi level, as its splitting induced by SOC can directly determine QSH gap. From Fig. 4(d), one can see that the ratio from the Pb- $p_{x, y}$ to bonded $\mathrm{X}$ orbital at the $\Gamma$ point near the Fermi level decreases in the order of $\mathrm{PbF}>\mathrm{PbCl}>\mathrm{PbBr}>\mathrm{PbI}$. Considering that the $\mathrm{Pb}$ exhibits stronger $\mathrm{SOC}$ strength than $\mathrm{X}$ atoms, it is expected that the larger the ratio is, the larger the contribution to the orbital near the Fermi level, and consequently the larger the SOC strength will be. As a result, we can understand why the global band gaps of these systems decrease in the contrary order of $\mathrm{PbF}>\mathrm{PbCl}>\mathrm{PbBr}>\mathrm{PbI}$.

The buckle structure generally sustain a larger strain than planar one, thus one might ask if these QSH insulators are robust under mechanical strain? In what follows, we investigate the effect of biaxial strain on topological properties of $\mathrm{PbX}$ monolayers. Here, we employ an in-plane strain on $\mathrm{PbX}$ maintaining the crystal symmetry by changing its lattices as $\varepsilon=\left(\mathrm{a}-\mathrm{a}_{0}\right) / \mathrm{a}_{0}$, where the $\mathrm{a}\left(\mathrm{a}_{0}\right)$ is strained (equilibrium) lattice constants. Figure 5 gives the variation of the direct gap $\left(E_{\mathrm{g}}(\Gamma)\right)$ at the $\Gamma$ point and indirect gap $\left(E_{\mathrm{g}}\right)$ with respect to external strain, which indicates the interatomic coupling can indeed modulate the topological properties. Especially, the QSH feature is rather robust when the strain falls into the range from -6 to $+8 \%$. Both the direct and indirect gaps decrease slightly with respect to tensile strain, but their nontrivial bulk-gaps within the strain range are still very large, i.e., larger than $0.96 \mathrm{eV}$ of $E_{\mathrm{g}}$ for $8 \%$ strain for $\mathrm{PbF}$ monolayer. Such robust topology paves the easy way for experimental realization. 


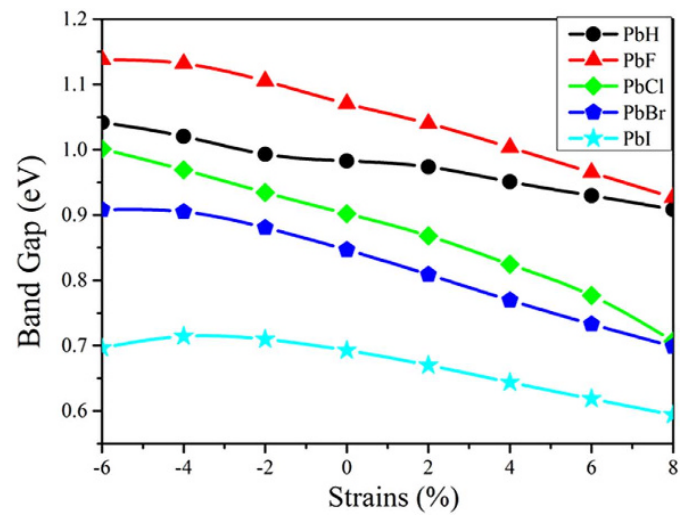

Figure 5. The calculated global band gap $\left(E_{\mathrm{g}}\right)$ of $\mathrm{PbX}$ with $\mathrm{SOC}$ as a function of external strain.

(a)
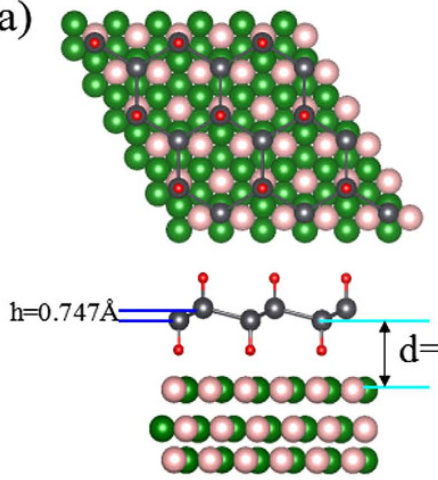

(c)

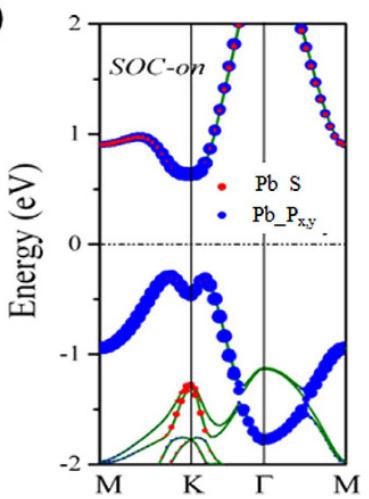

(b)
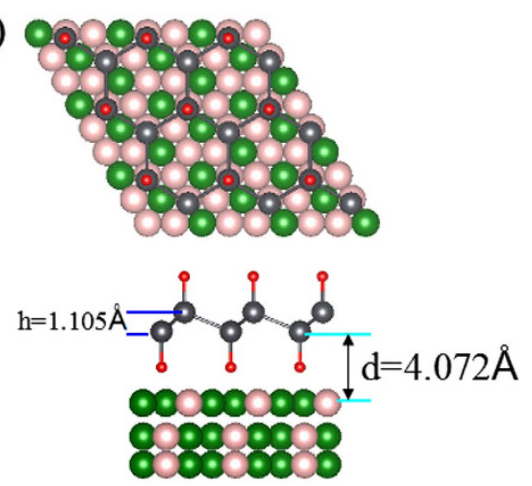

(d)

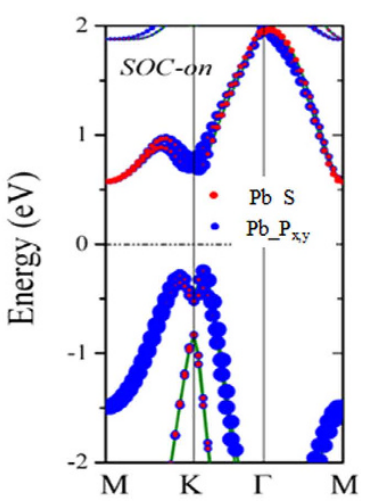

Figure 6. (a) Side and top views of the schematic illustration of the epitaxial growth $\mathrm{PbH}$ of large-gap QSH states on $2 \times 2 \mathrm{BN}$ substrate and (b) on $\sqrt{3} \times \sqrt{3}$ substrate. Orbital-resolved band structures with SOC based on DFT calculations. $(\mathbf{c}, \mathbf{d})$ notes the energy band of $\mathrm{PbH}$ on $2 \times 2$ and $\sqrt{3} \times \sqrt{3}$ BN substrate with SOC. The Fermi level is set to zero.

It is noticeable that, although the 2D QSH insulators have been extensively sought and studied theoretically and experimentally ${ }^{10-21}$, but few TIs with a bulk-gap exceeding $1.0 \mathrm{eV}$ has been discovered up to now. Here, a giant-gap as large as $1.34 \mathrm{eV}$ in $\mathrm{PbF}$ monolayer is the largest bulk-gap of all the reported 2D TIs, which are approximately four times the values of halogenated GeX/SnX films $(\sim 0.3 \mathrm{eV})$ and superstar 3D $\mathrm{Bi}_{2} \mathrm{Se}_{3}$ film $(\sim 0.35 \mathrm{eV})^{13}$. The topological states with a giant-gap are mainly due to the contribution of $p_{\mathrm{x}}$ and $p_{\mathrm{y}}$ orbitals of $\mathrm{Pb}$ atoms, instead of the next-nearest-neighbor SOC in the $\mathrm{p}_{\mathrm{z}}$ states $^{5,6}$. These giant bulk-gaps are advantageous for stabilizing the edge currents against the interference of thermally activated carriers in the bulk, which are beneficial to applications at room temperature.

On the experimental side, high-quality plumbene film in a honeycomb lattice has been successfully grown on different substrates based on the MBE method ${ }^{31}$. Also, a hydrogenated graphene and germanene ${ }^{32,33}$ are experimentally synthesized. Thus, it is expected that the PbX monolayers can also be fabricated or transferred on the substrate using similar techniques. As a $2 \mathrm{D}$ large-gap insulator with a high dielectric constant, $\mathrm{BN}$ sheet 
has been successfully used as the substrate to grow graphene or assembled in $2 \mathrm{D}$ stacked nanodevices ${ }^{34,35}$. Thus, we select the $\mathrm{BN}-\sqrt{3} \times \sqrt{3}(4.53 \AA)$ and $\mathrm{BN}-2 \times 2(5.23 \AA)$ as the substrate to support PbH monolayer. The substrate is modeled by placing 1-5 BN layers under $\mathrm{PbH}$ where all atoms in the bottom BN layer are fixed at bulk crystalline positions. To correctly describe the van der Waals interaction, we use a dispersion-corrected DFT method (optB88-vdW) ${ }^{36,37}$, which has been demonstrated to reliably describe 2D heterostructures. Figure $6(\mathrm{a}, \mathrm{b})$ show the optimized geometrical structures obtained by examining various positions of the $\mathrm{PbH}$ relative to $\mathrm{BN}$ substrate. We find that the lattice mismatch is only about $0.05 \%$ and $0.41 \%$ for the $\mathrm{BN}-\sqrt{3} \times \sqrt{3}$ and $\mathrm{BN}-2 \times 2$ substrates, while the distance between adjacent layers are $3.697 \AA$ and $3.578 \AA$, respectively. The binding energy is obtained to be $0.036 \mathrm{eV}$ per unit cell, indicating a weak interaction between $\mathrm{PbH}$ and $\mathrm{BN}$ sheet. As expected from the band structure in Fig. 6(c,d), $\mathrm{PbH}$ on the $\mathrm{BN}$ sheet remains semiconducting. Bader charge analysis indicates that there is essentially no charge transfer between adjacent layers, thus the states around the Fermi level are dominantly contributed by $\mathrm{PbH}$ monolayer. In comparison to the pristine $\mathrm{PbH}$, little difference is observed between them. Evidently, they are robust QSH insulators whose band inversion is not affected by the substrate.

In summary, based on first-principles calculations, we have predicted a new class of 2D QSH insulators in $\mathrm{PbX}$ monolayers with a giant-gap of $1.34 \mathrm{eV}$, allowing for viable applications at room temperature. This giant-gap opening is mainly due to the result of the strong SOC related to the $p_{x, y}$ orbitals of $\mathrm{Pb}$ atoms at the $\Gamma$ point, in sharp contrast to that consisting of the $p_{z}$ orbital as in pristine group-IV ones. The topological characteristic of $\mathrm{Pb}-\mathrm{H}$ monolayer is confirmed by the $\mathrm{Z}_{2}$ topological order due to $s-p_{x, y}$ band inversion and an explicit demonstration of the topological edge states, while the strong SOC enhances nontrivial gaps greatly. Also, these systems maintain their nontrivial topological phase within the certain strain range, indicating their topology is robust against strain. We also propose high-dielectric-constant $\mathrm{BN}$ as an ideal substrate for the experimental realization of $\mathrm{PbX}$, maintaining its nontrivial topology. These results represent a significant advance in the study of TIs and thus are expected to stimulate further work to synthesize, characterize and utilize $\mathrm{PbX}$ monolayers for fundamental exploration in spintronics.

\section{Methods}

First-principles calculations were performed by using density functional theory (DFT) methods as implemented in the Vienna ab initio simulation (VASP) ${ }^{38,39}$ package. The projector-augmented-wave potential, the Perdew-Burke-Ernzerhof (PBE) exchange-correlation functional ${ }^{40,41}$, was used to to treat the ion-electron interactions. The energy cutoff of the plane waves was set to $500 \mathrm{eV}$ with the energy precision of $10^{-6} \mathrm{eV}$. The Brillouin zone was sampled by using a $21 \times 21 \times 1$ Gamma-centered Monkhorst-Pack grid. The vacuum space was set to $20 \AA$ to minimize artificial interactions between neighboring slabs. SOC was included by a second variational procedure on a fully self-consistent basis. With the optimized structures, the more sophisticated HSE06 hybrid functional ${ }^{26}$ was used to check the corresponding results of the systems. The phonon spectra were calculated using a supercell approach within the PHONON $\operatorname{code}^{42}$.

\section{References}

1. Yan, B. \& Zhang, S. C. Topological Materials. Rep. Prog. Phys. 75, 096501-096523 (2012).

2. Moore, J. E. Majorana’s Wires. Nature nanotechnology. 8, 194-198 (2013).

3. Hasan, M. Z. \& Kane, C. L. Colloquium: Topological Insulators. Rev. Mod. Phys. 82, 3045-3067 (2010).

4. Qi, X. L. \& Zhang, S. C. Topological Insulators and Superconductors. Rev. Mod. Phys. 83, 1057-1110 (2011).

5. Kane, C. L. \& Mele, E. J. Quantum Spin Hall Effect in Graphene. Phys. Rev. Lett. 95, 226801 (2005).

6. Kane, C. L. \& Mele, E. J. Z 2 Topological Order and the Quantum Spin Hall Effect. Phys. Rev. Lett. 95, 146802 (2005).

7. Bernevig, B. A., Hughes, T. L. \& Zhang, S. C. Quantum Spin Hall Effect and Topological Phase Transition in HgTe Quantum Wells. Science. 314, 1757-1761 (2006).

8. König, M. et al. Quantum Spin Hall Insulator State in HgTe Quantum Wells. Science. 318, 766-770 (2007).

9. Du, L., Knez, I., Sullivan, G. \& Du, R. R. Robust Helical Edge Transport in Gated InAs/GaSb Bilayers. Phys. Rev. Lett. 114, 096802 (2015).

10. Liu, C., Hughes, T. L., Qi, X. L., Wang, K. \& Zhang, S. C. Quantum Spin Hall Effect in Inverted Type-II Semiconductors. Phys. Rev. Lett. 100, 236601 (2008).

11. Bernevig, B. A., Hughes, T. L. \& Zhang, S. -C. Quantum Spin Hall Effect and Topological Phase Transition in HgTe Quantum Wells. Science 314, 1757-1761 (2006).

12. Fu, L., Kane, C. L. \& Mele, E. J. Topological Insulators in Three Dimensions. Phys. Rev. Lett. 98, 106803-106806 (2007).

13. Zhang, H. et al. Topological Insulators in $\mathrm{Bi}_{2} \mathrm{Se}_{3}, \mathrm{Bi}_{2} \mathrm{Te}_{3}$ and $\mathrm{Sb}_{2} \mathrm{Te}_{3}$ with a Single Dirac Cone on the Surface. Nat. Phys. 5, 438-442 (2009).

14. Chen, Y. L. et al. Experimental Realization of a Three-Dimensional Topological Insulator, $\mathrm{Bi}_{2} \mathrm{Te}_{3}$. Science. 325, 178-181 (2009).

15. Soluyanov, A. A. \& Vanderbilt, D. Computing Topological Invariants Without Inversion Symmetry. Phys. Rev. B. 83, 235401-235410 (2011).

16. Si, C. et al. Functionalized Germanene as a Prototype of Large-gap Two-dimensional Topological Insulators. Phys. Rev. B. 89, 115429-115433 (2014)

17. Xu, Y. et al. Large-Gap Quantum Spin Hall Insulators in Tin Films. Phys. Rev. Lett. 111, 136804-136815 (2013).

18. Rivero, P., Yan, J. A., García-Suárez, V. M., Ferrer, J. \& Barraza-Lopez, S. Stability and Properties of High-buckled Two-dimensional Tin and Lead. Phys. Rev. B 90, 241408(R) (2014).

19. Jin, K. H. \& Jhi, S. H. Quantum Anomalous Hall and Quantum Spin-Hall Phases in Flattened Bi and Sb Bilayers. Sci. Rep. 5, 8426-8432 (2015).

20. Zhou, M. et al. Formation of Quantum Spin Hall State on Si Surface and Energy Gap Scaling with Strength of Spin Orbit Coupling. Sci. Rep. 4, 7102-7107 (2014).

21. Zhou, M. et al. Epitaxial Growth of Large-gap Quantum Spin Hall Insulator on Semiconductor Surface. PNAS 111, 14378-14381 (2014).

22. Zhang, R. W., Zhang, C. W., Ji, W. X., Li, S. S., Hu, S. J., Yan, S. S., Li, P., Wang, P. J. \& Li, F. Ethynyl-functionalized Stanene Film: a Promising Candidate as Large-gap Quantum Spin Hall Insulator. New J. Phys. 17, 083036 (2015).

23. Pumera, M. \& Wonga, C. H. A. Graphane and hydrogenated graphene. Chem. Soc. Rev. 42, 5987-5995 (2013).

24. Sofo, J. O., Chaudhari, A. S. \& Barber, G. D. Graphane: A Two-dimensional Hydrocarbon. Phy. Rev. B, 75, 153401 (2007). 
25. Tsai, W. F. et al. Gated Silicene as a Tunable Source of Nearly 100\% Spin-Polarized Electrons. Nat. Commun. 4, 1500 (2013).

26. Heyd, J., Scuseria, G. E. \& Ernzerhof, M. Hybrid Functionals Based on a Screened Coulomb Potential. J. Chem. Phys. 118, 8207-8215 (2003).

27. Mostofi, A. A. et al. Wannier 90: A Tool for Obtaining Maximally-Localised Wannier Functions. Comput. Phys. Commun. 178, 685-699 (2008).

28. Sancho, M. P. L., Sancho, J. M. L. \& Rubio, J. Quick Iterative Scheme for the Calculation of Transfer Matrices: Application to Mo (100). J. Phys. F: Met. Phys. 15, 851-858 (1985).

29. Fu, L. \& Kane, C. L. Topological Insulators with Inversion Symmetry. Phys. Rev. B. 76, 045302-045318 (2007).

30. Castro Neto, A. H. \& Guinea, F. Impurity-Induced Spin-Orbit Coupling in Graphene. Phys. Rev. Lett. 103, 026804 (2009).

31. Guo, Y. et al. Superconductivity Modulated by Quantum Size Effects. Science 306, 1915-1917 (2004)

32. Elias, D. C. et al. Control of Graphene's Properties by Reversible Hydrogenation: Evidence for Graphane. Science 323, 610-613 (2009).

33. Bianco, E. et al. Stability and Exfoliation of Germanane: a Germanium Graphane Analogue. ACS Nano 7, 4414-4421 (2013).

34. Britnell, L. et al. Field-Effect Tunneling Transistor Based on Vertical Graphene Heterostructures. Science 335, 947-950 (2012).

35. Ju, L. et al. Photoinduced Doping in Heterostructures of Graphene and Boron Nitride. Nat. Nanotechnol. 9, 348-352 (2014).

36. Klimeš, J., Bowler, D. R. \& Michaelides, A. Chemical Accuracy for the Van der Waals Density Functional. J. Phys. Condens. Matter. 22, 022201 (2010).

37. Klimeš, J., Bowler, D. R. \& Michaelides, A. Van der Waals Density Functionals Applied to Solids. Phys. Rev. B. 83, 195131 (2011).

38. Kresse, G. \& Furthmüller, J. Efficient Iterative Schemes for ab initio Total-energy Calculations Using a Plane-wave Basis Set. Phys. Rev. B. 54, 11169 (1996).

39. Kresse, G. \& Furthmüller, J. Efficiency of ab-initio Total Energy Calculations for Metals and Semiconductors Using a Plane-wave Basis Set. Comput. Mater. Sci. 6, 15-50 (1996).

40. Perdew, J. P., Burke, K. \& Ernzerhof, M. Generalized Gradient Approximation Made Simple. Phys. Rev. Lett. 77, 3865 (1996).

41. Blöchl, P. E. Projector Augmented-wave Method. Phys. Rev. B. 50, 17953 (1994).

42. Parlinski, K., Li, Z. Q. \& Kawazoe, Y. First-Principles Determination of the Soft Mode in Cubic ZrO 2 . Phys. Rev. Lett. 78, 4063-4066 (1997).

\section{Acknowledgements}

This work was supported by the National Natural Science Foundation of China (Grant No. 11274143, 11434006, 61172028, and 11304121), and Research Fund for the Doctoral Program of University of Jinan (Grant no. XBS1433).

\section{Author Contributions}

H.Z. and C.Z. conceived the study and wrote the manuscript. R.Z. and S.L. performed the first-principles calculations. W.J. calculated the phonon spectrum. P.L. and B.Z. prepared Figures 1-3, S.Y. and P.W. prepared Figures 4-6. All authors read and approved the final manuscript.

\section{Additional Information}

Supplementary information accompanies this paper at http://www.nature.com/srep

Competing financial interests: The authors declare no competing financial interests.

How to cite this article: Zhao, H. et al. Unexpected Giant-Gap Quantum Spin Hall Insulator in Chemically Decorated Plumbene Monolayer. Sci. Rep. 6, 20152; doi: 10.1038/srep20152 (2016).

(c) (i) This work is licensed under a Creative Commons Attribution 4.0 International License. The images or other third party material in this article are included in the article's Creative Commons license, unless indicated otherwise in the credit line; if the material is not included under the Creative Commons license, users will need to obtain permission from the license holder to reproduce the material. To view a copy of this license, visit http://creativecommons.org/licenses/by/4.0/ 